\title{
Investigation of Light Extraction Efficiency in AlGaN Deep Ultraviolet LEDs Using FDTD Simulations
}

\author{
Han-Youl Ryu* and Jong-In Shim** \\ * Department Physics, Inha University, Incheon 402-751, Korea \\ ** Department of Electronics and Communication Engineering, Hanyang University, Ansan 426-791, Korea
}

\begin{abstract}
Light extraction efficiency (LEE) in AlGaN deep ultraviolet (UV) light-emitting diodes (LEDs) is investigated using three-dimensional finite-difference time-domain simulations. For flip- chip LED structures, LEE is obtained to be $<\mathbf{1 0} \%$ due to strong $\mathrm{UV}$ light absorption in the $\mathrm{p}-\mathrm{GaN}$ layer. In addition, LEE of transverse-magnetic (TM) modes is found to be more than ten times smaller than that of transverse-electric (TE) modes, which explains the decreasing behavior of external quantum efficiency of $U V$ LEDs with decreasing wavelengths.
\end{abstract}

\section{INTRODUCTION}

Recently, deep ultraviolet (UV) light-emitting diodes (LEDs) based on AlGaN materials have drawn increasing attention for various applications such as water purification, sterilization, UV curing, and biomedical instrumentation [1-4]. However, despite rapid progress in increasing the performance of deep UV LEDs, the efficiency of AlGaN-based LEDs with wavelengths corresponding to the UV-C (200 to $280 \mathrm{~nm}$ ) range is still too low to justify the replacement of current UV lamps. The light extraction efficiency (LEE) of AlGaN-based deep UV LEDs has been thought to be quite low due to UV light absorption in the GaN p-contact layer. The low LEE is one of main limiting factor of the efficiency of AlGaN deep UV LEDs. However, there have been few quantitative studies on the LEE of AlGaN UV LEDs. In this research, we investigate the LEE of AlGaN-based deep UV LEDs using numerical simulations. A threedimensional (3-D) finite-difference time-domain (FDTD) method with a perfectly-matched-layer (PML) boundary condition is used for the simulation. Using the FDTD simulation, we calculate the LEE of representative deep UV LEDs with flip-chip structures.

\section{Simulation STRUCTURE}

The FDTD computational domains of the flip-chip UV LED structures for our LEE simulations are schematically drawn in Fig. 1. The layer structure of simulated LEDs is basically similar to that of recently reported deep UV LEDs [3,4]. On a sapphire substrate, an AlN buffer layer, an n-AlGaN layer, AlGaN multiplequantum-well (MQW) active layers, a p-AlGaN electron- blocking layer (EBL) layer, and a p-GaN contact layer are subsequently grown. The thickness of the AIN and the $\mathrm{n}$-AlGaN layer is assumed be 1 and $2 \mu \mathrm{m}$, respectively. A p-type electrode reflector exists below the p-GaN layer. For simplicity, the electrode reflector is assumed to be a perfect mirror with $100 \%$ reflectance. The absorption coefficient of the GaN layer, which has a strong influence on the LEE, is chosen to be $170,000 \mathrm{~cm}^{-1}[5,6]$. We assume that the LED chip was not encapsulated, and thus it is exposed to air.

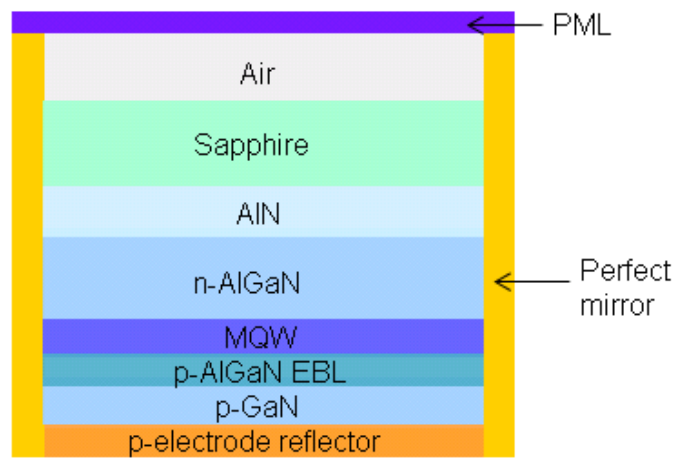

Fig. 1. Schematic side-view diagram of FDTD computational domains of flip-chip UV LED structures.

In the FDTD simulation, a single dipole source is positioned in the middle of the MQW active region. The peak wavelength and the full-width at half-maximum of the spectrum of light emitted from the MQWs is set at 280 and $10 \mathrm{~nm}$, respectively. The dipole source is polarized in the direction either parallel to the MQW plane for the excitation of the transverse-electric (TE) mode or perpendicular to the MQW plane for the excitation of the transverse-magnetic (TM) mode. The distance from the dipole source to the $\mathrm{p}-\mathrm{GaN}$ layer is fixed at $100 \mathrm{~nm}$. The TE- and the TM-polarized light propagates mainly in the vertical and the horizontal direction, respectively. Therefore, the LEE of the TMpolarized light should be much lower than that of the TEpolarized light.

\section{RESULTS AND DISCUSSION}

Fig. 2 presents our simulated results of the LEE for the 
FC LED structure shown in Fig. 1. The LEE of the TE and the TM mode is plotted as a function of the thickness of the p-GaN layer. As expected, the LEE of the TM mode is much lower than that of the TE mode for all $p$ $\mathrm{GaN}$ thicknesses considered. Depending on the $\mathrm{p}-\mathrm{GaN}$ thickness, the LEE of the TE mode varies from $4 \%$ to $15 \%$, whereas that of the TM mode varies from $0.2 \%$ to $1.1 \%$. When there is no $\mathrm{p}-\mathrm{GaN}$ layer, the LEE of the TE mode is still limited to be only $\sim 15 \%$, which is due to the total internal reflection of light inside the FC LED. The LEE for both modes varies periodically as the $\mathrm{p}-\mathrm{GaN}$ thickness increases up to $100 \mathrm{~nm}$. This periodic behavior results from the interference of upward emitted light and downward reflected light, and has also been observed in FC or micro-cavity LEDs operating in the visible wavelengths [7-9]. However, the LEE becomes nearly constant when the $\mathrm{p}-\mathrm{GaN}$ thickness is larger than $100 \mathrm{~nm}$ because light is almost completely absorbed in the $\mathrm{p}-\mathrm{GaN}$ layer for a $\mathrm{p}-\mathrm{GaN}$ thickness of $>100 \mathrm{~nm}$.

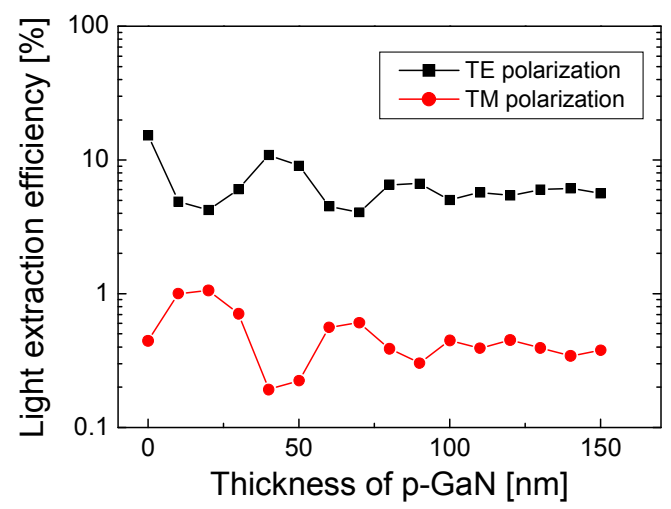

Fig. 2. Simulated results of light-extraction efficiency (LEE) for the FC LED structure shown in Fig. 1. LEE of the TE and the TM mode is plotted as a function of the thickness of the $\mathrm{p}-\mathrm{GaN}$ layer.

When the $\mathrm{p}-\mathrm{GaN}$ thickness is larger than $100 \mathrm{~nm}$, the LEE of the TE mode is $5 \%$ to $6 \%$ and that of the TM mode is $0.35 \%$ to $0.45 \%$. That is, the LEE of the TM mode is more than 10 times lower than that of the TE mode. This large difference in LEE between the TE and the TM mode implies that the LEE of UV LEDs may decrease considerably as the emission wavelength decreases. This is because the relative portion of the TM mode increases with decreasing wavelength. According to Refs. $[10,11]$, the relative portions of the TE mode and the TM mode become equal near $\sim 280 \mathrm{~nm}$. Then, by averaging the LEE of the TE and the TM mode, the LEE at a wavelength of $280 \mathrm{~nm}$ will only be $\sim 3 \%$ when the pGaN thickness is $>100 \mathrm{~nm}$. The EQE of the deep UV LEDs has been reported to decrease significantly as wavelength decreases, which has been mainly attributed to the decrease in the injection efficiency and the radiative efficiency with decreasing wavelengths. The results shown in Fig. 2 imply that the polarizationdependent LEE can also be responsible for the decrease in the LEE with decreasing wavelengths.

\section{Conclusions}

In this work, the LEE of an AlGaN-based deep UV LED with flip-chip structures was investigated based on FDTD simulations. It was proved that the FDTD method was quite effective to quantitatively evaluate the LEE of deep UV LEDs for each polarization mode. The LEE of deep UV LEDs was obtained to be $<10 \%$ at best due to strong UV light absorption in the $\mathrm{p}-\mathrm{GaN}$ contact layer. The LEE of the TM mode was found to be more than ten times lower than that of the TE mode. The presented simulation results are expected to provide some insight into understanding and hence increasing the LEE of AlGaN-based deep UV LEDs.

\section{REFERENCES}

[1] A. Khan, K. Balakrishnan, and T. Katona, "Ultraviolet light-emitting diodes based on group three nitrides," Nat. Photonics, vol. 2, p. 77, 2008

[2] M. S. Shur and R. Gaska, "Deep-ultraviolet light-emitting diodes," IEEE Trans. Electron Devices, vol. 57, p.12, 2010.

[3] H. Hirayama, "Recent progress of 220-280 nm-band AlGaN based deep-UV LEDs," Proc. SPIE, vol. 7617, p. 76171G-1, 2010.

[4] M. Kneissl, T. Kolbe, C. Chua, V. Kueller, N. Lobo, J. Stellmach, A. Knauer, H. Rodriguez, S. Einfeldt, Z. Yang, N. M. Johnson, and M. Weyers, "Advances in group IIInitride-based deep UV light-emitting diode technology," Semicond. Sci. Technol., vol. 26, p. 014036, 2011.

[5] G. Yu, G. Wang, H. Ishikawa, M. Umeno, T. Egawa, J. Watanabe, and T. Jimbo, "Optical properties of wurzite structure GaN on sapphire around fundamental edge by spectroscopic ellipsometry and the optical transmission method," Appl. Phys. Lett., vol. 70, p. 3209 (1997).

[6] E. F. Schubert, Refractive index and extinction coefficient of materials, p. 72, 2004.

[7] H. Benisty, H. De Neve, and C. Weisbuch, "Impact of planar microcavity effects on light extraction - Part I: Basic concepts and analytical trends," IEEE J. Quantum Electron., vol. 34, p. 1612, 1998.

[8] Y. C. Shen, J. J. Wierer, M. R. Krames, M. J. Ludowise, M. S. Misra, F. Ahmed, A. Y. Kim, G. O. Mueller, J. C. Bhat, S. A. Stockman, and P. S. Martin. "Optical cavity effects in $\mathrm{InGaN} / \mathrm{GaN}$ quantum-well-heterostructure flip-chip light-emitting diodes," Appl. Phys. Lett., vol. 82, p.2221, 2003.

[9] H. Y. Ryu and J. I. Shim, "Structural parameter dependence of light extraction efficiency in photonic crystal InGaN vertical light-emitting diode structures," IEEE J. Quantum Electron., vol. 46, p. 714, 2010.

[10] H. Kawanishi, E. Niikura, M. Yamamoto, and S. Takeda, "Experimental energy difference between heavy-or lighthole valence band and crystal-field split-off-hole valence band in AlGaN," Appl. Phys. Lett., vol. 89, p. 251107, 2006.

[11] T. Kolbe, A. Knauer, C. Chua, Z. Yang, S. Einfeldt, P. Vogt, N. M. Johnson, M. Weyers, and M. Kneissl, "Optical polarization characteristics of ultraviolet InAlGaN multiple quantum well light emitting diodes,” Appl. Phys. Lett., vol. 97, p. 171105, 2010. 